\title{
RECURSIVE LABELLING SYSTEMS AND STABILITY OF RECURSIVE STRUCTURES IN HYPERARITHMETICAL DEGREES
}

\author{
C. J. ASH
}

\begin{abstract}
We show that, under certain assumptions of recursiveness in $\mathfrak{A}$, the recursive structure $\mathfrak{A}$ is $\Delta_{\alpha}^{0}$-stable for $\alpha<\omega_{1}^{C K}$ if and only if there is an enumeration of $\mathfrak{A}$ using a $\Sigma_{\alpha}^{0}$ set of recursive $\Sigma_{\alpha}$ infinitary formulae and finitely many parameters from $\mathfrak{A}$. This extends the results of $[\mathbf{1}]$.

To do this, we first obtain results concerning $\Delta_{\alpha}^{0}$ paths in recursive labelling systems, also extending results of $[\mathbf{1}]$. We show, more generally, that a path and a labelling can simultaneously be defined, when each node of the path is to be obtained by a $\Delta_{\alpha}^{0}$ function from the previous node and its label.
\end{abstract}

Introduction. We say that a recursive structure $\mathfrak{A}$ is $\Delta_{\alpha}^{0}$-stable if, for every recursive structure $\mathfrak{B} \cong \mathfrak{A}$, every isomorphism from $\mathfrak{B}$ to $\mathfrak{A}$ is $\Delta_{\alpha}^{0}$ in Kleene's hyperarithmetical hierarchy. We shall show that, under certain assumptions, $\mathfrak{A}$ is $\Delta_{\alpha}^{0}$-stable iff there exists a "formally $\Delta_{\alpha}^{0}$ enumeration of $\mathfrak{A}$."

The basic outline of our argument is as in $[\mathbf{1}]$, where such a result was obtained for finite $\alpha$. However, certain technicalities prevent the generalization to infinite $\alpha$ from being as straightforward as might be expected. The argument seems most easily described in terms of recursive labelling systems, which were introduced in [1] for a similar purpose.

The two basic results for $\alpha$-systems are obtained in $\S 1$ and applied in the succeeding sections to the question of $\Delta_{\alpha}^{0}$-stability. These basic results are also used in the related topic of $\Delta_{\alpha}^{0}$-categoricity [2].

In $\S 1$ we define the notion of a recursively $\alpha$-guided recursive labelling system, or $\alpha$-system, and in Proposition 1 state the desired result similar to that of $[\mathbf{1}]$ involving the existence of r.e. points of $2^{\mathbf{N}}$ and of labellings of $\Delta_{\alpha}^{0}$ paths in an $\alpha$-system. To obtain this result for $a \geq \omega$, we need to deal with limit ordinals, and we frame an analogous result [Proposition 2] for $\left\langle\gamma_{n}\right\rangle$-systems where $\left\langle\gamma_{n}\right\rangle$ is an increasing sequence of ordinals. Propositions 1 and 2 are then established by the Main Lemma.

In $\S 2$ we show how the results of $\S 1$ apply to showing that structures are not $\Delta_{\alpha}^{0}$ stable, which is the "difficult direction" of our result. We show in Theorem 1 that under suitable conditions on $\mathfrak{A}$ there is a recursive structure $\mathfrak{B}$ and an isomorphism from $\mathfrak{B}$ to $\mathfrak{A}$ which is not $\Delta_{\alpha}^{0}$. In the case where $\alpha$ is a limit ordinal, we also give in Theorem 2 conditions under which there exists such a $\mathfrak{B}$ and an isomorphism which is not $\Delta_{\beta}^{0}$ for any $\beta<\alpha$.

Received by the editors June 18, 1984 and, in revised form September 19, 1985.

1980 Mathematics Subject Classification (1985 Revision). Primary 03D30. 
The method of proof for Theorems 1 and 2 is a little simpler than that given in $[\mathbf{1}]$. We comment that the method of $[\mathbf{1}]$ allows the assumptions of Theorems 1 and 2 to be slightly weakened.

In $\S 3$ we relate the results of $\S 2$ to recursive infinitary formulae. Theorem 4 shows that, under the conditions of Theorem 1 , a recursive structure $\mathfrak{A}$ is $\Delta_{\alpha}^{0}$-stable iff $\mathfrak{A}$ has a formally $\Delta_{\alpha}^{0}$ enumeration. Theorem 5 gives a similar result for the notion of $\tilde{\Delta}_{\alpha}^{0}$-stability introduced for Theorem 2 .

Theorem 4 can also be expressed in terms of the Scott rank of a structure. For a suitable notion of rank, we observe in Theorem 6 that a recursive structure $\mathfrak{A}$ satisfying the assumptions of Theorem 4 is $\Delta_{\alpha}^{0}$-stable iff $\mathfrak{A}$ has $<2^{\aleph_{0}}$ automorphisms and, for some finite $\bar{p},(\mathfrak{A}, \bar{p})$ has rank at most $\alpha$. Lastly in $\S 3$, we define $\mathfrak{A}$ to be Hyp-stable if for each recursive $\mathfrak{B} \cong \mathfrak{A}$, each isomorphism is hyperarithmetical. We note in Theorem 7 that we do not need methods such as those of $\S 1$ to deal with this notion because a recursive structure $\mathfrak{A}$ will be Hyp-stable iff it has fewer than $2^{\aleph_{0}}$ automorphisms. It follows that, without further assumptions, a recursive structure $\mathfrak{A}$ is Hyp-stable iff it is $\Delta_{\alpha}^{0}$-stable for some $\alpha<\omega_{1}^{C K}$.

Our main interest in this paper is the theoretical problem of finding general conditions under which a structure is not $\Delta_{\alpha}^{0}$-stable. The examples of conventional algebraic structures which we have considered do not provide examples of $\Delta_{\alpha}^{0}$ stability for $\alpha \geq \omega$. However, the notions of $\S \S 2$ and 3 may be illustrated by considering structures based on the ordinal numbers, such as $(\alpha,<)$ and $(\alpha,<,+)$. In $\S 4$ we summarize some of the results concerning the stability of such structures.

More details about recursion theory are to be found in [6] and about recursive structures in $[\mathbf{1}]$. We shall use Kleene's system 0 of ordinal notations and the related notions $<_{0},+_{0}$, and ||$_{0}$ [or just || ] which are described in $\S 11.7$ of [6]. The $\Delta_{\alpha}^{0}$ functions are as defined in $\S 16.8$ of $[6]$.

We use the phrase "recursive transfinite induction" to indicate Kleene's method for defining a partial recursive function on 0 . This consists of taking a partial recursive function $f$ and using the Recursion Theorem to obtain an $e$ for which $\phi_{e}(a)=f(e, a)$. The definition of $f(n, a)$ normally involves the restriction $\phi_{k(n, a)}$ of $\phi_{n}$ to $\left\{b: b<_{0} a\right\}$. If this definition guarantees that $f(n, a)$ is defined whenever $\phi_{k(n, a)}(b)$ is defined for all $b<_{0} a$, then it follows by classical transfinite induction on $|a|$ that $\phi_{e}(a)$ is defined for every $a \in 0$.

1. Recursive labelling systems. For applications in this paper, we are interested only in the metric space $2^{\mathbf{N}}$. But without much trouble we can obtain the results of this section for any suitably recursive complete metric space and so we shall do so.

DEFInition. A recursive metric space consists of a metric space $X$, a family $B(X)$ of nonempty open subsets of $X$, forming a basis for $X$, and an enumeration of $B(X)$ with respect to which the relations $\sigma \prec \tau$ [the closure of $\sigma$ is a subset of $\tau$ ] and $\delta(\sigma)<1 / n$ [the diameter of $\sigma$ is less than $1 / n$ ] are r.e. relations on $B(X) \times B(X)$ and $B(X) \times \mathbf{N}$, respectively.

Definition. An r.e. point of a recursive metric space $X$ is an element $x$ of $X$ such that $\{\sigma \in B(X): x \in \sigma\}$ is r.e.

An easily verified equivalent condition is that $\{x\}=\bigcap \sigma_{n}$ for some recursive sequence $\left\langle\sigma_{n}\right\rangle$ from $B(X)$ such that $\delta\left(\sigma_{n}\right) \rightarrow 0$ as $n \rightarrow \infty$. 
We note that, for the space $2^{\mathbf{N}}$ with its usual basis, the r.e. points are the recursive subsets of $\mathbf{N}$, not the r.e. subsets. This will be the case whenever $B(X)$ consists of clopen sets and the relation $\sigma \cap \tau=\varnothing$ on $B(X)$ is r.e., because then $x \notin \sigma$ iff, for some $\tau \in B(X), x \in \tau$ and $\sigma \cap \tau=\varnothing$.

By a complete recursive metric space, we mean a recursive metric space which is also complete in the classical sense, without further recursive assumptions. We need to use only the fact that if $\left\langle\sigma_{n}\right\rangle$ is a recursive sequence from $B(X), \delta\left(\sigma_{n}\right) \rightarrow 0$ and for each $n, \sigma_{n+1} \prec \sigma_{n}$, then $\bigcap \sigma_{n}=\{x\}$ where $x$ is an r.e. point. This follows since $x \in \sigma$ iff there exists $n$ for which $\sigma_{n} \prec \sigma$.

When discussing trees, we need to refer frequently to the levels of the nodes, so we give our definitions in terms of levels and of the predecessor function.

Definition. A tree $T$ consists of disjoint sets $\{a\}, T_{0}, T_{1}, \ldots$ and a predecessor function $p$ for which $p: T_{n+1} \rightarrow T_{n}, p: T_{0} \rightarrow\{a\}$, and, say, $p(a)=a$. If $p(x)=y$ then $y$ is the predecessor of $x$ and $x$ is a successor of $y$. The set of nodes of $T$ is the set $\{a\} \cup\left(\bigcup_{n} T_{n}\right)$. The apex of $T$ is the node $a$, and the nodes of $T$ of level $n$ are the elements of $T_{n}$.

[Thus no level is assigned to the element $a$. In fact, with our present conventions, the element $a$ plays no essential part in any construction and could be omitted.]

A recursive tree is one for which the set of nodes forms an r.e. set of natural numbers and the function $p$ is partial recursive. In this case, each set $T_{n}$ will be r.e., uniformly in $n$.

Since the function $p$ completely specifies $T$, we may define an index for a recursive tree to be an index for the partial recursive function $p$.

A path in $T$ is a sequence $u_{0}, u_{1}, \ldots$ [finite or infinite] for which $u_{n} \in T_{n}$ and $p\left(u_{n+1}\right)=u_{n}$.

A recursive labelling system on a recursive metric space $X$ is a quintuple $(T, L, S$, $N, F)$ for which $T$ is a recursive tree, $L$ is an r.e. set, $S$ is an r.e. subset of $T \times L$, $N$ is an r.e. subset of $T \times L \times T \times L$, and $F$ is a function from $L$ to subsets of $X$ for which the relation $F(l) \cap \sigma \neq \varnothing$ on $L \times B(X)$ is r.e.

A labelling of the path $u_{0}, u_{1}, \ldots$ in $T$ is a sequence $l_{0}, l_{1}, \ldots$ from $L$ of the same length such that, for each $n, S\left(u_{n}, l_{n}\right)$ and $N\left(u_{n}, l_{n}, u_{n+1}, l_{n+1}\right)$. An adherent point of a labelling $\left\langle l_{n}\right\rangle$ of an infinite path $\left\langle u_{n}\right\rangle$ is a point $x \in X$ such that, for every open set $U$ for which $x \in U$, there exists $N$ such that for all $n>N, F\left(l_{n}\right) \cap U \neq \varnothing$.

[Usually, for a correct labelling, we shall have $F\left(l_{0}\right) \supseteq F\left(l_{1}\right) \supseteq \cdots$, in which case $x \in X$ is adherent iff $x \in \bigcap_{n} \overline{F\left(l_{n}\right)}$. This will be the case for the $\alpha$-systems about to be considered whenever $\alpha>1$. The above definition of an adherent point removes, to some extent, the need to treat the case where $\alpha=1$ as a special case.]

We proceed to find sufficient conditions under which a nonrecursive path in $T$ nevertheless has a [nonrecursive] labelling with an r.e. adherent point. For some of our applications, however, such a result, generalizing that of $[\mathbf{1}]$, is not sufficient. We need also to consider situations where the desired path is determined at each level, not just by the previous node, but also by its label. We therefore make the following definitions.

DEFINITIONS. A path-generating function [or instruction] in a recursive labelling system is a function from $T \times L$ to $T$ mapping each $(u, l)$ for which $S(u, l)$ holds to some successor of $u$ in $T$. 
A labelling of an instruction, $p$, is an infinite path $u_{0}, u_{1}, \ldots$ in $T$ together with a labelling $l_{0}, l_{1}, \ldots$ of this path such that, for each $n, p\left(u_{n}, l_{n}\right)=u_{n+1}$.

We shall show that the existence of suitable relations on $L$ provides a sufficient condition for every $\Delta_{\alpha}^{0}$ instruction to have a $\Delta_{\alpha}^{0}$ labelling with an r.e. adherent point.

DEFinition. Let $\alpha<\omega_{1}^{C K}$ and let $\alpha=\beta+1$. A recursively $\alpha$-guided recursive labelling system [or $\alpha$-system] is a recursive labelling system $(T, L, S, N, F)$ together with a notation $a \in \mathcal{O}$ for $\alpha$ and a family $\left\{\triangleleft_{\gamma}: 1 \leq \gamma<\alpha\right\}$ of uniformly r.e. binary relations on $L$, indexed by $\left\{c: c<_{0} a\right\}$, for which conditions (1) to (7) below are satisfied. [Here $<_{0}$ denotes the partial ordering on $\mathcal{O}$, so that $\left\{c: c<_{0} a\right\}$ is an r.e. linearly ordered set of order type $\alpha$. Or course, we do not use in this instance the notation for 0 .]

(1) For each $u \in T_{0}$ and each $\sigma \in B(X)$ there exists $l \in L$ such that $S(u, l)$ and $F(l) \cap \sigma \neq \varnothing$.

(2) If $S(u, l)$ and $N(u, l, v, m)$, then $S(v, m)$.

(3) Each $\triangleleft_{\gamma}$ is reflexive and transitive.

(4) If $N(u, l, v, m)$, then $l \triangleleft_{\beta} m$.

(5) If $1 \leq \gamma_{1}<\gamma_{2}<\alpha$ and $l \triangleleft_{\gamma_{2}} m$, then $l \triangleleft_{\gamma_{1}} m$.

(6) If $l \triangleleft_{1} m$, then $F(l) \supseteq F(m)$.

(7) Suppose that $S(u, l), F(l) \cap \sigma \neq \varnothing$, and $v$ is a successor of $u$. [Suppose also that $\alpha>\alpha_{k}>\cdots>\alpha_{1}>\alpha_{0} \geq 1$ and that $l=l_{k} \triangleleft_{\alpha_{k}} \cdots \triangleleft_{\alpha_{2}} l_{1} \triangleleft_{\alpha_{1}} l_{0}$ where $\left.F\left(l_{0}\right) \cap \sigma \neq \varnothing.\right]^{*}$ Then there exists $m$ for which $N(u, l, v, m)$ and $F(m) \cap \sigma \neq \varnothing$ [and $l_{i} \triangleleft_{\alpha_{i}} m$ for $\left.i=0,1, \ldots, k\right]^{*}$.

${ }^{*}$ Notes. The two parts of condition (7) in parentheses are to be omitted in the cases where $\alpha=1$ or $\alpha=2$. In the case where $\alpha=1$, the sequence of $\triangleleft_{\gamma}$ 's is to be regarded as the empty sequence and only conditions (1), (2), and (7) are relevant. In the case where $\alpha=2$, we have $\beta=1$ and we need only the single relation $\triangleleft_{1}$, so condition (5) is irrelevant.

The following result will be established by the Main Lemma.

PROPOSITION 1. If $1 \leq \alpha<\omega_{1}^{C K}, \alpha$ is a successor ordinal and $\tau$ is an $\alpha$ system over a complete recursive metric space, then for every $\Delta_{\alpha}^{0}$ instruction in $T$ there is a $\Delta_{\alpha}^{0}$ labelling having an r.e. adherent point.

In order to prove this, we introduce similar notions for the case where $\alpha$ is a limit ordinal. In this case, we restrict our attention to certain particular classes of $\Delta_{\alpha}^{0}$ instructions.

DEFINITION. A special sequence for a limit ordinal $\alpha$ is an increasing sequence $\left\langle\gamma_{n}\right\rangle$ of successor ordinals whose limit is $\alpha$ and for which there is a recursive sequence $\left\langle c_{n}\right\rangle$ of notations from 0 such that $c_{0}<_{0} c_{1}<_{0} c_{2}<_{0} \cdots$.

In such a case, there will be a notation $a \in \mathcal{O}$ for $\alpha$ such that each $c_{n}<_{0} a$, namely $a=3.5^{e}$ where $e$ is an index for the recursive sequence $\left\langle c_{n}\right\rangle$. Conversely, if $\alpha<\omega_{1}^{C K}$ is any limit ordinal, then one may always obtain a special sequence $\left\langle\gamma_{n}\right\rangle$ for $\alpha$, for example by taking any notation $3.5^{e}$ for $\alpha$ and letting $\gamma_{n}=\left|\phi_{e}(n)+_{0} 1\right|$.

DEFINITIONS. Let $\alpha$ be a limit ordinal and let $\left\langle\gamma_{n}\right\rangle$ be a special sequence for $\alpha$.

A special $\Delta_{\alpha}^{0}$-instruction w.r.t. $\left\langle\gamma_{n}\right\rangle$ [or $\left\langle\gamma_{n}\right\rangle$-instruction] in a recursive labelling system is an instruction $p$ such that, for some recursive sequence $\left\langle e_{n}\right\rangle$, each $e_{n}$ is a $\Delta_{\gamma_{n}}^{0}$ index for the restriction of $p$ to $\left\{(u, l): u \in T_{n}\right\}$. 
A special $\alpha$-system w.r.t. $\left\langle\gamma_{n}\right\rangle$ [or $\left\langle\gamma_{n}\right\rangle$-system] is a recursive labelling system together with, first, a notation $a \in \mathcal{O}$ for $\alpha$, second a recursive sequence $\left\langle c_{n}\right\rangle$ of notations for the $\left\langle\gamma_{n}\right\rangle$, where $c_{0}<_{0} c_{1}<_{0} \cdots<_{0} a$, and third, a family $\left\{\triangleleft_{\gamma}: 1 \leq\right.$ $\gamma<\alpha\}$ of uniformly r.e. binary relations on $L$ indexed by $\left\{c: c<_{0} a\right\}$ which satisfy conditions (1), (2), (3), (5) and (6) above and conditions $(4)^{\prime}$ and (7)' below.

$(4)^{\prime}$ If $N(u, l, v, m)$ where $u \in T_{n}$ and if $\gamma_{n}=\beta_{n}+1$ where $\beta_{n}>0$, then $l \triangleleft_{\beta_{n}} m$.

$(7)^{\prime}$ Suppose that $S(u, l), \sigma \in B(X)$ and that $v$ is a successor of $u$ where $u \in T_{n}$. Suppose also that $\gamma_{n}>\alpha_{k}>\cdots>\alpha_{1}>\alpha_{0} \geq 1$ and that $l=l_{k} \triangleleft_{\alpha_{k}} \cdots \triangleleft_{\alpha_{2}} l_{1} \triangleleft_{\alpha_{1}} l_{0}$ where $F\left(l_{0}\right) \cap \sigma \neq \varnothing$. Then there exists $m$ for which $N(u, l, v, m), F(m) \cap \sigma \neq \varnothing$ and $l_{i} \triangleleft_{\alpha_{i}} m$ for $i=0,1, \ldots,(k-1)$.

For a $\left\langle\gamma_{n}\right\rangle$-instruction in a $\left\langle\gamma_{n}\right\rangle$-system, we may well expect a corresponding special sort of $\Delta_{\alpha}^{0}$ labelling.

DEFINITION. A $\left\langle\gamma_{n}\right\rangle$-labelling of an instruction $p$ in a recursive labelling system is a labelling $\left\langle u_{n}\right\rangle,\left\langle l_{n}\right\rangle$ of $p$ such that, for some recursive sequence $\left\langle e_{n}\right\rangle$, each $e_{n}$ is a $\Delta_{\gamma_{n}}^{0}$ index for the pair $\left(u_{n+1}, l_{n+1}\right)$.

The following result will also be established by the Main Lemma.

PROPOSITION 2. Let $\left\langle\gamma_{n}\right\rangle$ be a special sequence for a limit ordinal $\alpha$, where $\alpha<\omega_{1}^{C K}$, and let $\tau$ be a $\left\langle\gamma_{n}\right\rangle$-system over a complete recursive metric space. Then for every $\left\langle\gamma_{n}\right\rangle$-instruction in $T$ there is a $\left\langle\gamma_{n}\right\rangle$-labelling having an r.e. adherent point.

Comments on The MAIN Lemma. Before proceeding to the statement and proof of the Main Lemma, we comment that a more intuitive line of argument to establish Proposition 1 [and similarly Proposition 2] would be as follows.

Given an $\alpha$-system $\tau_{\alpha}$, we would construct a uniformly recursive sequence $\left\langle\tau_{\gamma}\right.$ : $1 \leq \gamma \leq \alpha\rangle$ for which each $\tau_{\gamma}$ is a $\gamma$-system in such a way that for each $\Delta_{\alpha}^{0}$ instruction $p_{\alpha}$ in $\tau_{\alpha}$ we could find a sequence $\left\langle p_{\gamma}: 1 \leq \gamma \leq \alpha\right\rangle$ where each $p_{\gamma}$ is a $\Delta_{\gamma}^{0}$ instruction in $\tau_{\gamma}$. These instructions would be arranged so that each $\Delta_{\gamma}^{0}$ labelling of $p_{\gamma}$ would determine a $\Delta_{\gamma+1}^{0}$ labelling of $p_{\gamma+1}$ having the same adherent points.

The $\tau_{\gamma}$ 's for which $\gamma$ is a limit ordinal would be special $\left\langle\gamma_{n}\right\rangle$-systems w.r.t. some special sequence $\left\langle\gamma_{n}\right\rangle$ for $\gamma$. Then $p_{\gamma}$ would be a $\left\langle\gamma_{n}\right\rangle$-instruction and a labelling of $p_{\gamma}$ would be obtained by referring, at the $(n+1)$ th level of $\tau_{\gamma}$, to the labelling of $p_{\gamma_{n}}$ in $\tau_{\gamma_{n}}$.

The path $p_{1}$ in $\tau_{1}$ would first be labelled to have an r.e. adherent point and then, proceeding by recursive transfinite induction, a $\Delta_{\gamma}^{0}$ labelling with the same adherent point would be obtained for each $p_{\gamma}$, in particular for $p_{\alpha}$.

A complication which arises in following precisely this outline, when $\alpha$ is infinite, is that each $\tau_{\gamma}$ must be defined in terms of later $\tau_{\gamma}$ 's and eventually in terms of $\tau_{\alpha}$. Thus, the definition of the $\tau_{\gamma}$ and similarly the $p_{\gamma}$ is not by recursive transfinite induction on $\gamma$. Rather, the systems $\tau_{\gamma}$ need not be defined simultaneously one level at a time. Thus the nodes of $\tau_{\gamma}$ of level $n$ and associated parts of $\tau_{\gamma}$ must be defined by recursive transfinite induction on some ordinal $\rho(\gamma, n)$. The function $\rho$ depends on some choice of a suitably nested family of special sequences for the limit ordinals $\gamma \leq \alpha$.

So it seems preferable, for the sake of examining the details carefully, to consider a slight generalization which can be proved more straightforwardly by recursive 
transfinite induction on $\alpha$. We no longer need to construct the sequence of systems $\tau_{\beta}$ explicitly, although this remains the intuitive guide and could be constructed by the same induction.

The generalization which we prove is that for each $\alpha$-system $\tau_{\alpha}$ and each $\beta<\alpha$ one can obtain a $\beta$-system $\tau_{\beta}$ for which the problem of labelling $\Delta_{\alpha}^{0}$ instructions in $\tau_{\alpha}$ is reducible to that of labelling $\Delta_{\beta}^{0}$ instructions in $\tau_{\beta}$. We make this precise:

DEFINITION. Let $\tau_{\alpha}$ be an $\alpha$-system and let $\beta<\alpha$. A $\beta$-precursor of $\tau_{\alpha}$ consists of:

(A) a $\beta$-system $\tau_{\beta}$,

(B) a partial recursive function which assigns to each index for a $\Delta_{\alpha}^{0}$-instruction $p_{\alpha}$ in $\tau_{\alpha}$ an index for a $\Delta_{\beta}^{0}$-instruction $p_{\beta}$,

(C) a partial recursive function which for each $p_{\beta}$ obtained from $p_{\alpha}$ as in (B) assigns to each index a $\Delta_{\beta}^{0}$ labelling of $p_{\beta}$ and index for a $\Delta_{\alpha}^{0}$ labelling of $p_{\alpha}$ having at least the same adherent points.

Note. In the case where $\alpha$ [or similarly $\beta$ ] is a limit ordinal, it is understood here that by a $\Delta_{\alpha}^{0}$-instruction or $\Delta_{\alpha}^{0}$-labelling we mean a $\left\langle\gamma_{n}\right\rangle$-instruction or $\left\langle\gamma_{n}\right\rangle$ labelling, where $\left\langle\gamma_{n}\right\rangle$ is the associated special sequence.

PROOF OF PROPOSITIONS 1 AND 2. If $\tau_{1}$ is a 1-precursor of an $\alpha$-system $\tau_{\alpha}$ and $p_{\alpha}$ is a $\Delta_{\alpha}^{0}$ instruction in $\tau_{\alpha}$, then the corresponding recursive instruction $p_{1}$ in $\tau_{1}$ may easily be recursively labelled, as in Lemma 17 of $[\mathbf{1}]$, to have an r.e. adherent point. Thus, Propositions 1 and 2 follow from the existence of precursors, to be proved in our Main Lemma.

MAIN LEMMA. If $\tau_{\alpha}$ is an $\alpha$-system, $\beta<\alpha$ and $\beta \leq \gamma_{0}$, in the case where $\alpha$ is a limit ordinal with special sequence $\left\langle\gamma_{n}\right\rangle$, then $\tau_{\alpha}$ has a $\beta$-precursor.

\section{PROOF.}

1. Method of proof. We define, by recursive transfinite induction on $a \in 0$, a recursive function which we call the precursor operator which assigns to each index for an $\alpha$-system using the notation $a$ for $\alpha$ and to each $b \in \mathcal{O}$ for which $b<_{0} a$ [and $b \leq_{0} c_{0}$ in the case where $\alpha$ is a limit ordinal and $\left\langle c_{n}\right\rangle$ is the sequence of notations for the special sequence] an index for a $\beta$-precursor, where $\beta=|b|$.

Thus it is sufficient to describe how to obtain a $\beta$-precursor $\tau_{\beta}$ of an $\alpha$-system $\tau_{\alpha}$ in terms of given precursors of $\gamma$-systems $\tau_{\gamma}$ for $\gamma<\alpha$.

We need to carry various properties of our precursors through the transfinite induction. The labels of the precursor of an $\alpha$-system and the associated definitions will be obtained from the corresponding notions in the $\alpha$-system. More generally

Definitions. An $\alpha$-set is a system $\mathcal{L}=\left(L, F, \triangleleft_{\gamma}\right)_{\gamma<\alpha}$ satisfying conditions (3), (5), and (6) of the definition of an $\alpha$-system.

A determining function for a $\beta$-system $\tau_{\beta}=\left(T_{\beta}, L_{\beta}, S_{\beta}, N_{\beta}, F_{\beta}, \triangleleft_{\gamma}^{\beta}\right)_{\gamma<\beta}$ is a function $g: L_{\beta} \rightarrow L$ for some $\alpha$-set $\mathcal{L}=\left(L, F, \triangleleft_{\gamma}\right)_{\gamma<\alpha}$, where $\alpha \geq \beta$ such that, for all $l \in L_{\beta}, F_{\beta}(l)=F(g(l))$ and, for all $l, m \in L_{\beta}, \gamma<\beta, l \triangleleft_{\gamma}^{\beta} m$ iff $g(l) \triangleleft_{\gamma} g(m)$.

We now add to the desired ingredients of a precursor, the inductive assumption that our precursor operation also yields:

(D) a determining function $g_{\alpha \beta}$ from $\tau_{\beta}$ to the $\alpha$-set of $\tau_{\alpha}$.

We also need to use the fact that our construction of a precursor of $\tau_{\alpha}$ is independent of those labels of $\tau_{\alpha}$ which are suitable for no nodes of $\tau_{\alpha}$. 
DEFINITION. Let $g$ be a determining function from an $\alpha$-system $\tau_{\alpha}$ to an $\alpha$-set $\mathcal{L}$, with the notation as above. We say that $\tau_{\alpha}$ is an $\alpha$-system w.r.t. $g$ if it satisfies the following strengthening of condition (7).

(7)* Suppose that $S_{\alpha}(u, l), F_{\alpha}(l) \cap \sigma=\varnothing$, and $v$ is a successor of $u$. [Suppose also that $\alpha>\alpha_{k}>\cdots>\alpha_{0} \geq 1$, that $g(l)=l_{k} \triangleleft_{\alpha_{k}} \cdots \triangleleft_{\alpha_{1}} l_{0}$, where $F\left(l_{0}\right) \cap \sigma \neq \varnothing$ and each $l_{i} \in L$ rather than $\left.L_{\alpha}\right]$. Then there exists $m \in L_{\alpha}$ for which $N_{\alpha}(u, l, v, m)$ and $F_{\alpha}(m) \cap \sigma \neq \varnothing\left[\right.$ and $l_{i} \triangleleft_{\alpha_{i}} g(m)$ for $\left.i=0,1, \ldots, k\right]$.

In the case where $\alpha$ is a limit ordinal, condition $(7)^{\prime}$ is modified in the same way.

We add to our inductive assumptions:

(E) If $g$ is any determining function for $\tau_{\alpha}$ such that $\tau_{\alpha}$ is an $\alpha$-system w.r.t. $g$, then $\tau_{\beta}$ is a $\beta$-system w.r.t. the composite $g \circ g_{\alpha \beta}$.

We need to use the fact that our precursors and the associated operations depend, at each level of the tree, only on this level and preceding levels of the corresponding features of the original $\alpha$-system.

Notation. For each $\gamma$-system $\tau$, let $\tau^{k}$ denote the system consisting of the nodes of $\tau$ of levels $\leq k$, the labels suitable for these nodes, and the restrictions to these nodes of $S, N$ and $\left\langle\triangleleft_{\xi}\right\rangle_{\xi<\gamma}$.

We add to our inductive assumptions:

(F) For $\alpha$-systems $\tau_{\alpha}$ and their $\beta$-precursors $\tau_{\beta}$, each $\tau_{\beta}^{k}$ depends only on $\tau_{\alpha}^{k}$. $\tau_{\alpha}^{k}$.

(G) Similarly, the restriction of the function $g_{\alpha \beta}$, in (D), to $\tau_{\beta}^{k}$ depends only on

(H) Likewise, the restriction of $p_{\beta}$, in $(\mathrm{C})$, to $\tau_{\beta}^{k}$ depends only on the restriction of $p_{\alpha}$ to $\tau_{\alpha}^{k}$.

COMMENT. So, in principle, the precursor operator acts on partial systems $\tau_{\alpha}^{k}$ to yield, in (A), the partial system $\tau_{\beta}^{k}$, in (D) a determining function from the labels of $\tau_{\beta}^{k}$ to those of $\tau_{\alpha}^{k}$ and, in (B) a partial recursive function acting on restriction of instructions $p_{\alpha}$ to $\tau_{\alpha}^{k}$. However, no such requirement is necessary [or indeed possible] for $(\mathrm{C})$.

We add further to our inductive assumptions two simplifications:

(I) The level 0 nodes and their labels are exactly the same for $\tau_{\beta}$ as for $\tau_{\alpha}$. The function $g_{\alpha \beta}$ is the identity function for such labels.

(J) The labelling of $p_{\alpha}$ given in (C) always has the same level 0 node with the same label as in the labelling of $p_{\beta}$.

Finally, we note that if $\gamma<\beta<\alpha, \tau_{\beta}$ is a $\beta$-precursor of $\tau_{\alpha}$ and $\tau_{\gamma}$ is the $\gamma$ precursor of $\tau_{\beta}$, then $\tau_{\gamma}$ is a suitable choice for a $\gamma$-precursor of $\tau_{\alpha}$, the associated operations being obtained by composition. It is therefore sufficient to describe how to obtain a $\beta$-precursor of an $\alpha$-system $\tau_{\alpha}$ first when $\alpha=\beta+1$ [for which we consider the two cases where $\beta$ is or is not a limit ordinal] and then when $\alpha$ is a limit ordinal and $b=c_{0}$, where $\left\langle c_{n}\right\rangle$ is the associated sequence of notations. We now do this in parts 2,3 , and 4 below.

In the case where $\alpha=\beta+1$ and $\beta$ is a limit ordinal, in order to obtain a $\gamma$ precursor for $\tau_{\alpha}$ where $\gamma<\beta$, we must obtain a $\gamma$-precursor for the $\beta$-precursor of $\tau_{\alpha}$. Thus, the special sequence $\left\langle\gamma_{n}\right\rangle$ for $\beta$ must have $\gamma \leq \gamma_{0}$. We therefore show in this case that any special sequence for $\beta$ can be used. [This is why, in our definitions, we did not restrict attention only to the fundamental sequence given by the notation for $\beta$.] 
2. The case $\alpha=\beta+1$ where $\beta$ is a successor ordinal. Let $\tau_{\alpha}=\tau=$ $\left(T, L, S, N, F, \triangleleft_{\gamma}\right)_{\gamma<\alpha}$. If $\alpha=\beta+1$, we let the $\beta$-precursor of $\tau$ be the system $\tau^{\prime}=\left(T^{\prime}, L^{\prime}, S^{\prime}, N^{\prime}, F^{\prime}, \triangleleft_{\gamma}^{\prime}\right)_{\gamma<\beta}$ defined as follows.

The tree $T^{\prime}$ consists of all the sequences $\left(u_{0}, u_{1}, \ldots, u_{n}\right)$ from $T$ for which each $u_{k}$ has level $k$ in $T$ and each $u_{k+1}$ is a finite successor of $u_{0}$. The predecessor of $\left(u_{0}, u_{1}, \ldots, u_{n}\right)$ is $\left(u_{0}, u_{1}, \ldots, u_{n-1}\right)$.

If $u=\left(u_{0}, u_{1}, \ldots, u_{n}\right)$ and the path in $T$ with last node $u_{n}$ is $r_{0}, r_{1}, \ldots, r_{n}$ [so $\left.r_{n}=u_{n}\right]$, then we define $S^{\prime}(u, l)$ to hold when $l=\left(l_{0}, l_{1}, \ldots, l_{n}\right)$ is a labelling in $T$ of this path. The set $L^{\prime}$ consists of all such sequences $l$. The determining function $g$ for $\tau^{\prime}$ is defined by $g(l)=l_{n}$, and $F^{\prime}$ and the $\triangleleft_{\gamma}^{\prime}$ are defined according to the definition of a detrmining function. When all else is done, we may replace $\left(u_{0}\right)$ and $\left(l_{0}\right)$ by $u_{0}$ and $l_{0}$ for the sake of assumptions $(\mathrm{I})$ and $(\mathrm{J})$.

For $u, v \in T^{\prime}, l, m \in L^{\prime}$, we define $N^{\prime}(u, l, v, m)$ as follows. Let $u=\left(u_{0}, u_{1}, \ldots\right.$, $\left.u_{n}\right)$. We require that $v=\left(u_{0}, u_{1}, \ldots, u_{n}, u_{n+1}\right)$ for some $u_{n+1}$ and that $S^{\prime}(u, l)$ and $S^{\prime}(v, m)$. Thus $l=\left(l_{0}, l_{1}, \ldots, l_{n}\right)$ and $m=\left(m_{0}, m_{1}, \ldots, m_{n}, m_{n+1}\right)$ are labellings in $\tau$ of paths $r_{0}, \ldots, r_{n}$ and $r_{0}, \ldots, r_{a-1}, s_{1}, \ldots, s_{n+1}$, where $r_{n}=u_{n}$ and either $s_{a} \neq r_{a}$ or $a=n+1$. Then we let $N^{\prime}(u, l, v, m)$ hold if, additionally, $m_{0}=l_{0}$, $m_{1}=l_{1}, \ldots, m_{a-1}=l_{a-1}$, and $l_{n} \triangleleft_{\gamma} m_{n+1}$ where $\beta=\gamma+1$. We note that, here, $a \geq 1$, by the definition of $T^{\prime}$.

Conditions (1) to (6) for a $\beta$-system are straightforward. To see that $\tau^{\prime}$ is a $\beta$-system w.r.t. $g$ also satisfying assumption (E), suppose that $\tau$ is an $\alpha$-system w.r.t. some determining function $h$ from $\tau$ to an $\alpha$-set $\left(L^{+}, F^{+}, \triangleleft_{\gamma}^{+}\right)_{\gamma<\alpha}$. We must verify condition $(7)^{*}$ for $\tau^{\prime}$ and $h \circ g$.

Suppose, then, that $v$ is a successor of $u$ in $T^{\prime}, l \in L^{\prime}, S^{\prime}(u, l), \beta>\alpha_{k}>$ $\cdots>\alpha_{0} \geq 1$, and that $h(g(l))=l_{k}^{+} \triangleleft_{\alpha_{k}}^{+} \cdots \triangleleft_{\alpha_{1}}^{+} l_{0}^{+}$, where each $l_{i}^{+} \in L^{+}$and $F^{+}\left(l_{0}^{+}\right) \cap \sigma \neq \varnothing$. If $\beta=\gamma+1$, then we may assume that $\alpha_{k}=\gamma$, since $l_{k}^{+} \triangleleft_{\gamma}^{+} l_{k}^{+}$. Using the notation above, we must find a labelling $m=\left(l_{0}, \ldots, l_{a-1}, m_{a}, \ldots, m_{n+1}\right)$ of the path $r_{0}, \ldots, r_{a-1}, s_{a}, \ldots, s_{n+1}$ in $\tau$.

Since $l=\left(l_{0}, \ldots, l_{n}\right)$ is a labelling of a path in the $\alpha$-system $T$, we have $l_{a-1} \triangleleft_{\beta} l_{n}$ and so $h\left(l_{a-1}\right) \triangleleft_{\beta}^{+} h\left(l_{n}\right)=l_{k}^{+} \triangleleft_{\alpha_{k}}^{+} l_{k-1}^{+} \cdots \triangleleft_{\alpha_{1}}^{+} l_{0}^{+}$. Now by condition $(7)^{*}$ for $\tau$ and $h$, we may find $m_{a} \in L$ for which $N\left(r_{a-1}, l_{a-1}, s_{a}, m_{a}\right), F\left(m_{a}\right) \cap \sigma \neq \varnothing$, $h\left(l_{a-1}\right) \triangleleft_{\beta}^{+} h\left(m_{a}\right)$, and $l_{i}^{+} \triangleleft_{\alpha_{i}} h\left(m_{a}\right)$ for $0 \leq i \leq k$. We may then choose the remaining $m_{i}$ in turn using (7) for $\tau$, so that $F\left(m_{n+1}\right) \cap \sigma \neq \varnothing$. Then, since $h\left(l_{n}\right)=l_{k}^{+} \triangleleft_{\alpha_{k}}^{+} h\left(m_{a}\right)$ and $\alpha_{k}=\gamma$, we have $l_{n} \triangleleft_{\gamma} m_{a} \triangleleft_{\beta} m_{n+1}$, so $l_{n} \triangleleft_{\gamma} m_{n+1}$ and $N^{\prime}(u, l, v, m)$. For $0 \leq i \leq k$, since $l_{i}^{+} \triangleleft_{\alpha_{i}}^{+} h\left(m_{a}\right)$ and $m_{a} \triangleleft_{\beta} m_{n+1}$, we have $h\left(m_{a}\right) \triangleleft_{\beta}^{+} h\left(m_{n+1}\right)$ and so $l_{i}^{+} \triangleleft_{\alpha_{i}}^{+} h\left(m_{n+1}\right)=h(g(m))$, as required.

For condition (B), given any $\Delta_{\alpha}^{0}$ instruction $p$ in $\tau$, we obtain a corresponding $\Delta_{\beta}^{0}$ instruction $p^{\prime}$ in $\tau^{\prime}$ as follows. Since $\alpha=\beta+1$, we may let $p(u, l)=\lim _{s} \hat{p}(u, l, s)$ where $\hat{p}$ is a $\Delta_{\beta}^{0}$ function. For $u^{\prime} \in T^{\prime}, l^{\prime} \in L^{\prime}$ such that $S^{\prime}\left(u^{\prime}, l^{\prime}\right)$, let $u^{\prime}=$ $\left(u_{0}, \ldots, u_{n}\right)$ and $l^{\prime}=\left(l_{0}, \ldots, l_{n}\right)$. Then $l^{\prime}$ is a labelling of the path $r_{0}, \ldots, r_{n}$ in $\tau$, where $r_{n}=u_{n}$. We consider the least $i$ for which $i=n$ or $\hat{p}\left(r_{i}, l_{i}, n\right) \neq r_{i+1}$, and let $p^{\prime}\left(u^{\prime}, l^{\prime}\right)$ be $\left(u_{0}, \ldots, u_{n}, u_{n+1}\right)$ where $u_{n+1}$ is any level $(n+1)$ node of $T$ which is a finite successor of $\hat{p}\left(r_{i}, l_{i}, n\right)$.

Then, for condition (C), suppose that $\left\langle u_{n}^{\prime}\right\rangle$ and $\left\langle l_{n}^{\prime}\right\rangle$ form a labelling of $p^{\prime}$ in $\tau^{\prime}$. Let $u_{n}^{\prime}=\left(u_{n 0}, u_{n 1}, \ldots, u_{n n}\right), l_{n}^{\prime}=\left(l_{n 0}, l_{n 1}, \ldots, l_{n n}\right)$, and let $r_{n 0}, r_{n 1}, \ldots, r_{n n}$ be the path in $\tau$ for which $r_{n n}=u_{n n}$. We may then see, by induction on $n$, that 
for each $n$ the limits $u_{n}=\lim _{s} r_{s n}$ and $l_{n}=\lim _{s} l_{s n}$ exist and that $\left\langle u_{n}\right\rangle$ and $\left\langle l_{n}\right\rangle$ constitute a labelling of $p$ in $\tau$. If $x$ is an adherent point of the labelling $\left\langle l_{n}^{\prime}\right\rangle$ and $U$ is an open set where $x \in U$, there exists $N$ such that $F^{\prime}\left(l_{n}^{\prime}\right) \cap U \neq \varnothing$ for all $n>N$. Then for $s \geq n>N$ we have $l_{s n} \triangleleft_{\beta} l_{s s}$, so $F\left(l_{s n}\right) \supseteq F\left(l_{s s}\right)=F^{\prime}\left(l_{s}^{\prime}\right)$ and so, for $n>N$, we have $F\left(l_{n}\right) \cap U \neq \varnothing$. Thus $x$ is also an adherent point of $\left\langle l_{n}\right\rangle$.

3. The case $\alpha=\beta+1$ where $\beta$ is a limit ordinal. Let $\left\langle\gamma_{n}\right\rangle$ be any special squence for $\beta$. Then we may obtain a $\left\langle\gamma_{n}\right\rangle$-system as a $\beta$-precursor of $\tau_{\alpha}=\tau$ in very much the same way as in the previous case. The only modifications needed are the following.

In the definition of $N^{\prime}(u, l, v, m)$, the stipulation $l_{n} \triangleleft_{\gamma} m_{n+1}$ where $\beta=\gamma+1$ is replaced by $l_{n} \triangleleft_{\beta_{n}} m_{n+1}$ where $\gamma_{n}=\beta_{n}+1$. In the verification that $\tau^{\prime}$ is a $\left\langle\gamma_{n}\right\rangle$-system satisfying condition (E), $\beta$ and $\gamma$ are replaced by the appropriate $\gamma_{n}$ and $\beta_{n}$, respectively.

To obtain a $\left\langle\gamma_{n}\right\rangle$-instruction $p^{\prime}$ for $\tau^{\prime}$ corresponding to the instruction $p$ for $\tau$, we first obtain a recursive sequence $\left\langle e_{n}\right\rangle$ where $e_{n}$ is an index for a $\Delta_{\gamma_{n}}^{0}$ function $p_{n}(u, l)$ and such that $p(u, l)=\lim _{n} p_{n}(u, l)$. The instruction $p^{\prime}$ is now obtained in the same way as before except that $\hat{p}(u, l, n)$ is replaced by $p_{n}(u, l)$.

4. The case where $\alpha$ is a limit ordinal. Let

$$
\tau_{\alpha}=\tau=\left(T, L, S, N, F,<_{\gamma}\right)_{\gamma<\alpha}
$$

be an $\alpha$-system and let $\left\langle\gamma_{n}\right\rangle$ be the associated special sequence for $\alpha$. We must construct a $\gamma_{0}$-precursor of $\tau$. We do this by constructing a sequence $\tau_{0}, \tau_{1}, \ldots$, where each $\tau_{n}$ is a $\gamma_{n}$-system. Then $\tau_{0}$ will be the desired $\gamma_{0}$-precursor of $\tau$.

The $\tau_{n}$ are defined simultaneously as follows. The level 0 nodes of $\tau_{n}$ are exactly the level $n$ nodes of $\tau$, with the same suitable labels. The level $k+1$ nodes of $\tau_{n}$ and their suitable labels are those of level $k$ of the $\gamma_{n}$-precursor $\tau_{n+1}^{\prime}$ of $\tau_{n+1}$. By assumption (I), the level 0 nodes of $\tau_{n+1}^{\prime}$ are those of $\tau_{n+1}$ and so are level $n+1$ nodes of $\tau$. Thus we may define the predecessor in $\tau_{n}$ of each level 1 node to be as in $\tau$. For nodes of $\tau_{n}$ of levels greater than 1 , the predecessors are as in $\tau_{n+1}^{\prime}$.

In this way, each $\tau_{n}$ is defined in terms of $\tau$ and $\tau_{n+1}$. However, the definition is justified since each $\tau_{n}^{k+1}$ depends only on $\tau^{n}$ and $\left(\tau_{n+1}^{\prime}\right)^{k}$ and, by assumption (F), $\left(\tau_{n+1}^{\prime}\right)^{k}$ depends only on $\tau_{n+1}^{k}$. Thus the $\tau_{n}^{k}$ are well defined by induction on $k$. [Moreover, each $\tau_{n}^{k}$ depends ultimately only on $\tau^{k+n}$, so $\tau_{0}^{k}$ depends only on $\tau^{k}$, as required by condition (F) for a precursor.] The other ingredients of our construction are defined by the same form of induction.

Each $\tau_{n}$ will have a determining function, $g_{n}$, to the $\alpha$-set of $\tau$. The set, $L_{n}$, of labels for $\tau_{n}$ consists of the labels of $\tau$ suitable for level $n$ nodes of $\tau$, on which $g_{n}$ is the identity function, together with the labels of $\tau_{n+1}^{\prime}$ on which $g_{n}$ is $g_{n+1} \circ h_{n}$, where $h_{n}$ is the determining function from $\tau_{n+1}^{\prime}$ to $\tau_{n+1}$.

The relations $S_{n}(u, l)$ and $N_{n}(u, l, v, m)$ for $\tau_{n}$ are defined to be as in $\tau$ if $u$ has level 0 and as in $\tau_{n+1}^{\prime}$ otherwise. The function $F$ and the relations $\left\langle\triangleleft_{\gamma}^{n}\right\rangle_{\gamma<\gamma_{n}}$ are defined in the unique way which makes $g_{n}$ a determining function for $\tau_{n}$.

To show that $\tau_{0}$ is a $\gamma_{0}$-system w.r.t. $g_{0}$ and satisfies condition (E), we suppose that $\tau$ is an $\alpha$-system w.r.t. a determining function $h$ to an $\alpha$-set $\mathcal{L}^{+}=$ $\left(L^{+}, F^{+}, \triangleleft_{\gamma}^{+}\right)_{\gamma<\alpha}$ and prove simultaneously that each $\tau_{n}$ is a $\gamma_{n}$-system w.r.t. $h \circ g_{n}$.

The arguments are, in principle, by induction on the largest level of labels or nodes involved in each condition for a $\gamma_{n}$-system. The effect of this is that we 
may show that $\tau_{n}$ is a $\gamma_{n}$-system w.r.t. $h \circ g_{n}$ under the assumption that $\tau_{n+1}$ is a $\gamma_{n+1}$-system w.r.t. $h \circ g_{n+1}$. Conditions (1) to (6) follow immediately from the definitions.

For condition $(7)^{*}$ for $\tau_{n}$ and $h \circ g_{n}$, suppose that $S_{n}(u, l), F_{n}(l) \cap \sigma \neq \varnothing$, and $v$ is a successor of $u$ in $\tau_{n}$. Suppose also that $\alpha>\alpha_{k}>\cdots>\alpha_{0} \geq 1$ and that $h\left(g_{n}(l)\right)=l_{k} \triangleleft_{\alpha_{k}}^{+} \cdots \triangleleft_{\alpha_{1}}^{+} l_{0}$ where $F^{+}\left(l_{0}\right) \cap \sigma \neq \varnothing$ and each $l_{i} \in L^{+}$.

First, if $u$ has level 0 , then $u, v \in T, g_{n}(l)=l$, and, by $(7)^{*}$ for $\tau$ and $h$, there exists $m \in L$ such that $N(u, l, v, m), F(m) \cap \sigma \neq \varnothing$ and, for $0 \leq i \leq k, l_{i} \triangleleft_{\alpha_{i}}^{+} h(m)$. But then, by our definitions, $m \in L_{n}, N_{n}(u, l, v, m), F_{n}(m) \cap \sigma=F\left(g_{n}(m)\right) \cap \sigma=$ $F(m) \cap \sigma \neq \varnothing$ and, for $0 \leq i \leq k, l_{i} \triangleleft_{\alpha_{i}}^{+} h(m)=h\left(g_{n}(m)\right)$.

Now suppose that $u$ has level greater than 0 . Then $u, v$ are nodes of $\tau_{n+1}^{\prime}=$ $\left(T_{n+1}^{\prime}, L_{n+1}^{\prime}, S_{n+1}^{\prime}, N_{n+1}^{\prime}, F_{n+1}^{\prime}, \triangleleft_{\gamma}^{\prime n}\right)_{\gamma<\gamma_{n}}$. By our assumption, $\tau_{n+1}$ is a $\gamma_{n+1}$ system w.r.t. $h \circ g_{n+1}$, so by the transfinite induction hypothesis $\tau_{n+1}^{\prime}$ is a $\gamma_{n^{-}}$ system w.r.t. $h \circ g_{n+1} \circ h_{n}$. Thus there exists $m \in L_{n+1}^{\prime}$ such that $N_{n+1}^{\prime}(u, l, v, m)$, $F_{n+1}^{\prime}(m) \cap \sigma \neq \varnothing$ and, for $1 \leq i \leq k, l_{i} \triangleleft_{\alpha_{i}}^{+} h\left(g_{n+1}\left(h_{n}(m)\right)\right)=h\left(g_{n}(m)\right)$.

For (B), suppose that $p$ is a $\left\langle\gamma_{n}\right\rangle$-instruction for $\tau$. We obtain a corresponding $\Delta_{\gamma_{0}}^{0}$ instruction $p_{0}$ for $\tau_{0}$ by simultaneously defining for each $n$ a $\Delta_{\gamma_{n}}^{0}$ instruction $p_{n}$ for $\tau_{n}$. On level 0 nodes of $\tau_{n}, p_{n}$ acts as $p$ does on level $n$ nodes of $\tau$. On other nodes of $\tau_{n}$, we let $p_{n}$ act as does $p_{n+1}^{\prime}$, where $p_{n+1}^{\prime}$ is the instruction for $\tau_{n+1}^{\prime}$ assigned to the instruction $p_{n+1}$ for $\tau_{n+1}$ according to $(\mathrm{B})$ of the inductive hypothesis.

For (C), suppose that $\lambda_{0}$ is a $\Delta_{\gamma_{0}}^{0}$ labelling of $p_{0}$ in $\tau_{0}$. We obtain by straightforward induction on $n$ a $\Delta_{\gamma_{n}}^{0}$ labelling $\lambda_{n}$ of each $p_{n}$ having the same adherent points. Having obtained $\lambda_{n}$, we obtain $\lambda_{n+1}$ as follows. We note that $\lambda_{n}$ consists of a level 0 node, $u_{n}$, of $\tau_{n}$ and a label, $l_{n}$, for this, followed by a $\Delta_{\gamma_{n}}^{0}$ labelling $\lambda_{n+1}^{\prime}$ of $p_{n+1}^{\prime}$ in $\tau_{n+1}^{\prime}$. Part (C) of the inductive hypothesis for $\tau_{n+1}$ thus yields a labelling $\lambda_{n+1}$ of $p_{n+1}$. The sequence of first nodes, $u_{n}$, of the $\lambda_{n}$ and their labels, $l_{n}$, then form [using assumption $(\mathrm{J})$ for the $\tau_{n+1}$ ] a labelling $\lambda$ of $p$ in $\tau$.

If $x$ is an adherent point of $\lambda_{0}$, then it is so for each $\lambda_{n}$, by (C) of the inductive hypothesis, and so certainly for $n \geq 1$ we have $x \in \bigcap \overline{F_{n}(l)}$ for each label $l$ occurring in $\lambda_{n}$. In particular, $x \in \bigcap \overline{F_{n}\left(l_{n}\right)}$ for $n \geq 1$, so $x$ is an adherent point of $\lambda$.

This concludes the proof.

2. Conditions for instability. Our constructions depend on the "back-andforth" relations $\leq_{\beta}$ between finite sequences, of the same length, of elements of a structure $\mathfrak{A}$, where $\beta$ is an ordinal number.

DEFINITION. For each structure $\mathfrak{A}$ we define $\leq_{\beta}$ by transfinite induction on $\beta \geq 1$. We define $\bar{a} \leq_{1} \bar{b}$ if each finitary universal formula true for $\bar{a}$ in $\mathfrak{A}$ is true for $\bar{b}$. We define $\bar{a} \leq_{\beta+1} \bar{b}$ if, for each sequence $\bar{d}$ there exists a sequence $\bar{c}$ such that $\bar{a}, \bar{c} \geq_{\beta} \bar{b}, \bar{d}$. If $\delta$ is a limit ordinal, we define $\bar{a} \leq_{\beta} \bar{b}$ if $\bar{a} \leq_{\beta} \bar{b}$ for all $\beta<\delta$.

DEFInITION. For each structure $\mathfrak{A}$, each finite sequence $\bar{p}$ from $\mathfrak{A}$ and each ordinal $\alpha \geq 2$, we define the subset $\operatorname{cl}_{\alpha}(\bar{p})$ of $A$ as follows. If $\alpha=\beta+1$, then $x \in \operatorname{cl}_{\alpha}(\bar{p})$ if for some $\bar{a}$, whenever $\bar{p}, x, \bar{a} \leq_{\beta} \bar{p}, x^{\prime}, \bar{a}^{\prime}$, then $x=x^{\prime}$. If $\delta$ is a limit ordinal then $\operatorname{cl}_{\delta}(\bar{p})=\bigcap_{\beta<\delta} \operatorname{cl}_{\beta}(\bar{p})$.

In all the simple examples where $\mathfrak{A}$ is $\Delta_{\alpha}^{0}$-stable we have $A=\operatorname{cl}_{\alpha}(\bar{p})$ for some $\bar{p}$. We can prove this in general, provided that we make certain additional assumptions about the recursiveness of $\mathfrak{A}$. 
THEOREM 1. Let $\mathfrak{A}$ be a recursive structure such that for no $\bar{p}$ is $A=\operatorname{cl}_{\alpha}(\bar{p})$. Then $\mathfrak{A}$ is not $\Delta_{\alpha}^{0}$-stable whenever the following conditions are satisfied.

(1) The existential diagram of $\mathfrak{A}$ is recursive.

(2) For some notation a for $\alpha$, the relations $\leq_{\beta}$ on $\mathfrak{A}$ for $1 \leq \beta<\alpha$ are uniformly r.e. when indexed by $\left\{b: b<_{0} a\right\}$.

(3) There is a recursive procedure which chooses, for each $\bar{p}$, an element $x \in A$ for which $x \notin \mathrm{cl}_{\alpha}(\bar{p})$.

Proof. If the domain, $A$, of $\mathfrak{A}$ is finite, then clearly $A=\operatorname{cl}_{\alpha}(\bar{p})$ where $A=\{\bar{p}\}$. So we may suppose that $A=\left\{a_{0}, a_{1}, \ldots\right\}$ and let $B=\left\{b_{0}, b_{1}, \ldots\right\}$ be any recursive set. Let $L$ be the language for $\mathfrak{A}$ and let $\left\{\theta_{0}, \theta_{1}, \ldots\right\}$ be an enumeration of the atomic sentences of $L(B)$. We obtain a recursive structure $\mathfrak{B}$ whose domain is $B$ by enumerating, during our construction, the atomic diagram $D(\mathfrak{B})$ of $\mathfrak{B}$, or, in the terminology of $\S 1$, by obtaining an r.e. point $x$ of the metric space $X=2^{\mathbf{N}}$ with the usual basis as $B(X)$, which yields $D(\mathfrak{B})$ by

$$
\begin{cases}\theta_{k} \in D(\mathfrak{B}) & \text { if } x(k)=1, \\ \neg \theta_{k} \in D(\mathfrak{B}) & \text { if } x(k)=0 .\end{cases}
$$

Thus, the nonempty basic open sets $\sigma \in B(X)$ correspond to consistent finite subsets $\Sigma(\sigma)$ of $\left\{\theta_{k}: k \in \mathbf{N}\right\} \cup\left\{\neg \theta_{k}: k \in \mathbf{N}\right\}$.

Let $P$ be the set of all finite partial one-one functions from $B$ to $A$. We define $f \in P$ to be coherent w.r.t. $\sigma \in B(X)$ if there is a bijection $g \supseteq f$ from $B$ to $A$ such that, for each $\theta\left(b_{i_{1}}, \ldots, b_{i_{k}}\right) \in \Sigma(\sigma)$ we have $\mathfrak{A} \vDash \theta\left[g\left(b_{i_{1}}\right), \ldots, g\left(b_{i_{k}}\right)\right]$. It follows from assumption (1) that this is a recursive relation between $f$ and $\sigma$.

The relation $\triangleleft_{\beta}$ needed to apply the results of $\S 1$ are obtained from similar relations on $P$ defined as follows.

Definition. For $f, g \in P$ and $\beta \geq 1$, let $f \leq_{\beta} g$ if $\operatorname{dom}(f) \subseteq \operatorname{dom}(g)$ and $a_{1}, \ldots, a_{n} \leq_{\beta} b_{1}, \ldots, b_{n}$, where $\left\{a_{1}, \ldots, a_{n}\right\}=\operatorname{ran}(f)$ and each $b_{i}=g\left(f^{-1}\left(a_{i}\right)\right)$.

We may now prove the following [similarly to Lemmas $3,4,5,6,7$ of $[\mathbf{1}]$ and using the definition of $\mathrm{cl}_{\alpha}$ ].

LEMMA 1. If $f \in P$ is coherent w.r.t. $\sigma \in B(X)$ then for each $\bar{a} \in A, \bar{b} \in B$, and for each $n=1,2, \ldots$ there exists $g \in P$ and $\sigma^{\prime} \in B(X)$ for which $g \supseteq f$, $\bar{a} \in \operatorname{ran}(g), \bar{b} \in \operatorname{dom}(g), \delta\left(\sigma^{\prime}\right)<1 / n$, and $g$ is coherent w.r.t. $\sigma^{\prime}$.

LEMMA 2. If $f \triangleleft_{\beta} g$ and $g$ is coherent w.r.t. $\sigma$, then $f$ is coherent w.r.t. $\sigma$.

LEMMA 3. If $f \leq_{\alpha} g, g$ is coherent w.r.t. $\sigma, g(y)=x$, and $x \notin \operatorname{cl}_{\alpha}(\operatorname{ran}(f))$, then for each $\beta<\alpha$ there exists a coherent $h \supseteq f$ for which $g \triangleleft_{\beta} h$ and $h(y) \neq x$.

LEMMA 4. If $\alpha_{k}>\cdots>\alpha_{1}>\alpha_{0} \geq 1$ and $f_{k} \triangleleft_{\alpha_{k}} f_{k-1} \triangleleft_{k-1} \cdots \triangleleft_{\alpha_{2}} f_{1} \triangleleft_{\alpha_{1}} f_{0}$ where $f_{0}$ is coherent w.r.t. $\sigma \in B(X)$, then there exists $g \supseteq f_{k}$, coherent w.r.t. $\sigma$, such that $f_{i} \triangleleft_{\alpha_{i}} g$ for $i=1,2, \ldots,(k-1)$.

To resume the proof, we define an $(\alpha+1)$-system as follows. The nodes of the tree of level $n$ consist of sequences of the form $\left(\mu_{0}\left(m_{0}\right)=k_{0}, \ldots, \mu_{n-1}\left(m_{n-1}\right)=\right.$ $\left.k_{n-1}\right)$ where the $\mu_{e}$ are symbols corresponding to the partial functions $\phi_{e}^{a}$ for some notation $a$ for $\alpha$. The predecessor function is the usual one for sequences, that is, the function which deletes the last term of a sequence. In this tree there is, as well as the apex, a single node of level 0 , namely the empty sequence. A suitable 
label for such a node is a pair $(f, x)$ for which $f \in P, x \in A$, and $x \notin \operatorname{cl}_{\alpha}(\operatorname{ran}(f))$. A correct next label on the successor node $\left(\mu_{0}\left(m_{0}\right)=k_{0}, \ldots, \mu_{n}\left(m_{n}\right)=k_{n}\right)$ is a suitable label $(g, y)$ such that $g \subseteq f, a_{n}, x \in \operatorname{ran}(g), b_{n} \in \operatorname{dom}(g)$ and if $m_{n}=x$, then $g^{-1}(x) \neq k_{n}$. For each label $(f, x), F(f, x)$ is defined to be the set of points of $2^{\mathbf{N}}$ which correspond to structures $\mathfrak{B}$ for which there is an isomorphism from $\mathfrak{B}$ to $\mathfrak{A}$ extending $f$. This means that $F(f, x) \cap \sigma \neq \varnothing$ iff $f$ is coherent w.r.t. $\sigma$.

To show that this is an $\alpha+1$-system, we define $(f, x) \triangleleft_{\beta}(g, y)$ if $f \triangleleft_{\beta} g$, in the case where $\beta<\alpha$, and $(f, x) \triangleleft_{\alpha}(g, y)$ if $f \subseteq g$. Clause (7) of the definition then requires that, given $\alpha \geq \alpha_{k}>\cdots>\alpha_{1}>\alpha_{0} \geq 1, f_{k} \triangleleft_{\alpha_{k}} \cdots \triangleleft_{\alpha_{2}} f_{1} \triangleleft_{\alpha_{1}} f_{0}, \sigma \in B(X)$, and $x \notin \mathrm{cl}_{\alpha}\left(\operatorname{ran}\left(f_{k}\right)\right)$, where $f_{0}$ is coherent w.r.t. $\sigma$, there exists $g \supseteq f_{k}$ for which $g$ is coherent w.r.t. $\sigma, x, a_{n} \in \operatorname{ran}(g), b_{n} \in \operatorname{dom}(g), f_{i} \triangleleft_{\alpha_{i}} g$ for $i=0, \ldots,(k-1)$ and $g^{-1}(x) \neq k$. By Lemma 4 there exists a coherent $h$ for which $h \supseteq f_{k}$ and $f_{i} \triangleleft_{\alpha_{i}} h$ for $i=0, \ldots,(k-1)$. By Lemma 1 we may choose a coherent $h_{1} \supseteq h$ for which $x \in \operatorname{ran}(h)$, say $x=h(y)$. By Lemma 3 there exists a coherent $h_{2} \supseteq f_{k}$ for which $h_{1} \triangleleft_{\alpha_{k-1}} h_{2}$ [since $\alpha_{k-1}<\alpha$ ] and $h_{2}(y) \neq x$. By Lemma 1 again, we may choose a coherent $g \supseteq h_{2}$ for which $a_{n}, x \in \operatorname{ran}(g)$ and $b_{n} \in \operatorname{dom}(g)$. So $f_{k} \subseteq h_{2} \subseteq g$ and thus $f_{k} \subseteq g$, and for $i<k, f_{i} \triangleleft_{\alpha_{i}} h \subseteq h_{1} \triangleleft_{\alpha_{k-1}} h_{2} \subseteq g$ and thus $f_{i} \triangleleft_{\alpha_{i}} g$.

The desired instruction is that which proceeds from a node of level $n$ with label $(f, x)$ to the successor node $\left(\ldots, \mu_{n}(x)=k\right)$ for which

$$
k= \begin{cases}\phi_{n}^{a}(x) & \text { if this is defined } \\ 0 & \text { (say) otherwise. }\end{cases}
$$

Clearly this is a $\Delta_{\alpha+1}^{0}$ instruction and so, by Proposition 1, there is a correct labelling $\left(g, x_{0}\right),\left(f_{0}, x_{1}\right),\left(f_{1}, x_{2}\right), \ldots$ of this instruction having an r.e. adherent point. Let $\mathfrak{B}$ be the structure determined by this point and let $f=\bigcup_{n} f_{n}$. Then $f: \mathfrak{B} \cong \mathfrak{A}$ and for each $e$, if $\phi_{e}^{n}$ is total then $f_{e}^{-1}\left(x_{e}\right) \neq \phi_{e}^{a}\left(x_{e}\right)$. Hence $f$ is not $\Delta_{\alpha}^{0}$.

A logical extension of the notion of stability arises from considering a recursive structure $\mathfrak{A}$ which is $\Delta_{\alpha}^{0}$-stable, where $\alpha$ is a limit ordinal, but for each $\beta<\alpha$ is not $\Delta_{\beta}^{0}$-stable. Then, for each $\beta<\alpha$ there exists $\mathfrak{B}_{\beta} \cong \mathfrak{A}$ such that no isomorphism from $\mathfrak{B}_{\beta}$ to $\mathfrak{A}$ is $\Delta_{\beta}^{0}$. We ask whether a single such $\mathfrak{B}$ can be chosen independently of $\beta$.

Definition. A recursive structure $\mathfrak{A}$ is $\hat{\Delta}_{\alpha}^{0}$-stable, where $\alpha$ is a limit ordinal, if for each recursive $\mathfrak{B} \cong \mathfrak{A}$ and each isomorphism $f: \mathfrak{B} \cong \mathfrak{A}$ there exists $\beta<\alpha$ for which $f \in \Delta_{\beta}^{0}$. [Thus $\hat{\Delta}_{\alpha}^{0}$ represents the class $\bigcup_{\beta<\alpha} \Delta_{\beta}^{0}$.]

THEOREM 2. Suppose that $\alpha$ is a limit ordinal and that, for each $\beta<\alpha$ and each $\bar{p} \in A, \operatorname{cl}_{\beta}(\bar{p}) \neq A$. Then $\mathfrak{A}$ is not $\hat{\Delta}_{\alpha}^{0}$-stable provided that the following conditions are satisfied.

(1) The existential diagram of $\mathfrak{A}$ is recursive.

(2) For some notation a for $\alpha$, the relations $\leq_{\beta}$ for $1 \leq \beta<\alpha$ are uniformly r.e. when indexed by $\left\{b: b<_{0} a\right\}$.

(3) For the same notation a for $\alpha$, there is a recursive procedure which chooses, for each $\bar{p}$, uniformly in a notation $b<_{0}$ a for $\beta$, an element $x \in A$ for which $x \notin \mathrm{cl}_{\beta}(\bar{p})$.

PROOF. We use the same terminology as for the proof of Theorem 1. Let $a$ be the notation for $\alpha$ given in assumptions (2) and (3), and let $\left\langle d_{n}\right\rangle$ be a recursive 
sequence of notations for ordinals $\left\langle\beta_{n}\right\rangle$ such that $d_{0}<_{0} \quad d_{1}<_{0} \cdots<_{0} \quad a$ and $\lim _{n} \beta_{n}=\alpha$. For simplicity we may assume that $\beta_{0} \geq 2$. Let $\gamma_{n}=\beta_{n}+1$.

We define a special $\alpha$-system w.r.t. $\left\langle\gamma_{n}\right\rangle$ as follows. The nodes of the tree of level $n$ are those sequences of the form

$$
\left(\mu_{(0)_{0}}^{(0)_{1}}\left(m_{0}\right)=k_{0}, \ldots, \mu_{(n-1)_{0}}^{(n-1)_{1}}\left(m_{n-1}\right)=k_{n-1}\right)
$$

where the $\mu_{e}^{r}$ are symbols corresponding to the partial functions $\phi_{e}^{d_{r}}$ and $n=$ $\left\langle(n)_{0},(n)_{1}\right\rangle$ is a recursive pairing function for which $(n)_{1} \leq n$.

A suitable label for such a node is a pair $(f, x)$ for which $f \in P, x \in A$ and $x \notin \operatorname{cl}_{\beta_{n}}(\operatorname{ran}(f))$. A correct next label on the successor node $\left(\ldots, \mu_{(n)_{0}}^{(n)_{1}}\left(m_{n}\right)=k_{n}\right)$ is a suitable label $(g, y)$ such that $g \supseteq f, a_{n}, x \in \operatorname{ran}(g), b_{n} \in \operatorname{dom}(f)$, and if $m_{n}=x$, then $g^{-1}(x) \neq k_{n}$. The function $F$ is again defined so that $F(f, x) \cap \sigma \neq \varnothing$ if and only if $f$ is coherent w.r.t. $\sigma$.

For $\beta<\alpha$, we define $(f, x) \triangleleft_{\beta}(g, y)$ if $f \triangleleft_{\beta} g$. The verification that the result is a $\left\langle\gamma_{n}\right\rangle$-system is much as for the proof of Theorem 1 .

The desired instruction is that which proceeds from a node of level $n$ to the successor node $\left(\ldots, \mu_{(n)_{0}}^{(n)_{1}}(x)=k\right)$ for which

$$
k= \begin{cases}\phi_{(n)_{0}}^{d_{(n)_{1}}}(x) & \text { if this is defined } \\ 0 & \text { (say) otherwise }\end{cases}
$$

Since $d_{(n)_{1}}<_{0} c_{0}$, we can find a $\Delta_{c_{n}}^{0}$ index for this value of $k$, so we have defined a $\left\langle\gamma_{n}\right\rangle$-instruction. By Proposition 2, there is a correct labelling of this having an r.e. adherent point giving, as in the previous proof, a recursive structure $\mathfrak{B}$ and an isomorphism $f: \mathfrak{B} \cong \mathfrak{A}$ which is not $\Delta_{\beta_{n}}^{0}$ for any $n$.

COMMENTS ON THE PROOFS. Theorem 1 could instead be proved as in [1] using "binary patterns" as labels for an $\alpha$-system. The proof given here seems simpler and, incidentally, shows that we could equally construct a recursive $\mathfrak{B}$ and an isomorphism not in any uniform family of total $\Delta_{\alpha+1}^{0}$ functions. On the other hand, the method of [1] shows that the conditions of Theorem 1 can be weakened to those given in [1]. Assumption (3) can be replaced by:

$(3)^{\prime}$ There is a $\Delta_{2}^{0}$ function which assigns to each $\bar{p}$ an element of $A-\operatorname{cl}_{\alpha}(\bar{p})$.

A more complicated argument, following the proof of the Main Lemma, shows that Theorem 2 can similarly be modified, replacing assumption (3) by

$(3)^{\prime}$ There is a $\Delta_{2}^{0}$ function which yields, for each $\bar{p}$ and each notation $b<_{0} a$ for $\beta$, an element of $A-\operatorname{cl}_{\beta}(\bar{p})$.

3. Infinitary formulae. We define the $\Sigma_{\alpha}$ and $\Pi_{\alpha}$ formulae of $L_{\omega_{1} \omega}$ as follows. The $\Sigma_{0}$ and $\Pi_{0}$ formulae are the quantifier-free formulae of $L_{\omega \omega}$. The $\Sigma_{\alpha+1}$ formulae are those of the form $\bigvee_{n \in S} \exists \bar{y}_{n} \phi_{n}$ where each $\phi_{n}$ as in $\Pi_{\alpha}$ formula and $\bar{y}_{n}$ is a finite sequence of variables. The $\Pi_{\alpha+1}$ formulae are those of the form $\bigwedge_{n \in S} \forall \bar{y}_{n} \phi_{n}$ where each $\phi_{n}$ is a $\Sigma_{\alpha}$ formula. If $\delta$ is a limit ordinal, the $\Sigma_{\delta}$ formulae are $\bigvee_{n \in S} \phi_{n}$ where each $\phi_{n}$ is $\Sigma_{\beta}$ for some $\beta<\delta$ and the $\Pi_{\delta}$ formulae are $\Lambda_{n \in S} \phi_{n}$ where each $\phi_{n}$ is $\Pi_{\beta}$ for some $\beta<\delta$.

For $a \in 0$, we define the (recursive) $\Sigma_{a}$ and $\Pi_{a}$ formulae and simultaneously, by recursive transfinite induction, their Gödel numbers. If $a=2^{b}$ and if, for each $n \in W_{e}, \phi_{e}(n)=\left\langle i_{n}, j_{n}\right\rangle$ where $i_{n}$ is a Gödel number for a $\Pi_{b}$ formula $\theta_{n}$ and $j_{n}$ 
is a canonical index for a finite sequence of variables $\bar{y}_{n}$, then $\langle a, e, 0\rangle$ is a Gödel number for the $\Sigma_{a}$ formula $\bigvee_{n \in W_{e}} \exists \bar{y}_{n} \theta_{n}$. Similarly, if instead each $i_{n}$ is a Gödel number of a $\Sigma_{b}$ formula $\theta_{n}$, then $\langle a, e, 1\rangle$ is a Gödel number for the $\Pi_{a}$ formula $\bigwedge_{n \in W_{e}} \forall \bar{y}_{n} \theta_{n}$. If $a=3.5^{k}$ where $\phi_{k}(n)=a_{n}$ and if, for each $n, \phi_{e}(n)$ is a Gödel number for a $\Sigma_{a_{n}}$ formula $\theta_{n}$, then $\langle a, e, 0\rangle$ is a Gödel number for the $\Sigma_{a}$ formula $\bigvee_{n \in W_{e}} \theta_{n}$. If, instead, each $\theta_{n}$ is $\Pi_{a_{n}}$, then $\langle a, e, 1\rangle$ is a Gödel number for the $\Pi_{a}$ formula $\bigwedge_{n \in W_{e}} \theta_{n}$.

We define a recursive $\Sigma_{a}$ formula to be one which is $\Sigma_{a}$ for some $a \in \mathcal{O}$ such that $|a|=\alpha$. We note that, similarly to $\S 16.8$ of $[1]$, if $|b| \leq|a|$ then we can effectively find, from a Gödel number of a $\Sigma_{b}$ formula, a Gödel number of a logically equivalent $\Sigma_{a}$ formula.

We make the following definition as seeming a natural condition under which $\mathfrak{A}$ will be $\Delta_{\alpha}^{0}$-stable.

DEFinition. A formally $\Delta_{\alpha}^{0}$ enumeration of $\mathfrak{A}$ consists of a finite sequence $\bar{p}$ from $A$, an ordinal notation $a \in \mathcal{O}$ for $\alpha$ and a $\Sigma_{\alpha}^{0}$ set $S$ of Gödel numbers for a set of recursive $\Sigma_{a}$ formulae such that, if $\phi_{n}$ denotes the formula with Gödel number $n$, then, for each $n \in S, \mathfrak{A} \vDash \phi_{n}[c, \bar{p}]$ for at most one element $c$ of $A$ and for each element $c$ of $A$ there exists at least one $n \in S$ such that $\mathfrak{A} \vDash \phi_{n}[c, \bar{p}]$.

COMMENT. Given a formally $\Delta_{\alpha}^{0}$ enumeration of $\mathfrak{A}$, we may convert it into one for which $S$ is recursive and such that, for each $n \in S, \mathfrak{A} \vDash \phi_{n}[c, \bar{p}]$ for exactly one $c \in A$.

It is easy to see that the existence of such an enumeration is a sufficient condition for $\Delta_{\alpha}^{0}$-stability.

THEOREM 3. If a recursive structure $\mathfrak{A}$ has a formally $\Delta_{\alpha}^{0}$ enumeration, then $\mathfrak{A}$ is $\Delta_{\alpha}^{0}$-stable.

PROOF. First we note that, by recursive transfinite induction, from a Gödel number for a recursive $\Sigma_{a}$ formula, we can compute an index for the $\Sigma_{\alpha}^{0}$ relation which it determines on a recursive structure.

Now suppose that $\mathfrak{B}$ is a recursive structure and $f: \mathfrak{B} \cong \mathfrak{A}$. Then for some $\bar{q} \in B$ we have $f:(\mathfrak{B}, \bar{q}) \cong(\mathfrak{A}, \bar{p})$ and $f(c)=d$ iff there exists $n \in S$ for which $\mathfrak{A} \vDash \phi_{n}[c, \bar{p}]$ and $\mathfrak{B} \vDash \phi_{n}[d, \bar{q}]$. But this relation is $\Sigma_{\alpha}^{0}$, and so $f$ is $\Delta_{\alpha}^{0}$.

The results of $\S 2$ allow us to prove the converse of Theorem 3 under certain assumptions. First, the following may be proved, substantially as for Lemmas 1 and 2 of $[\mathbf{1}]$.

LEMMA 5. Suppose that the relations $\leq_{\beta}$ on $\mathfrak{A}$ for $1 \leq \beta<\alpha$ are uniformly r.e. when indexed by $\left\{b: b<_{0} a\right\}$ for some $a \in \mathcal{O}$ with $|a|=\alpha$ and that the existential diagram of $\mathfrak{A}$ is recursive. Then, from each $\bar{c} \in A$, we can effectively find a Gödel number for a recursive $\Pi_{a}$ formula $\phi_{\bar{c}}^{a}$ such that, for all $\bar{d} \in A, \bar{c} \leq_{\alpha} \bar{d}$ iff $\mathfrak{A} \vDash \phi_{\bar{c}}^{a}[\bar{d}]$.

LEMMA 6. Under the same assumptions, if, for some $\bar{p}, \operatorname{cl}_{\alpha}(\bar{p})=A$, then $\mathfrak{A}$ has a formally $\Delta_{\alpha}^{0}$ enumeration.

Thus, the results of Theorems 1 and 3 may be combined as follows, using Lemma 6. We incorporate the improvements stated in $\S 2$.

THEOREM 4. Suppose that the existential diagram of $\mathfrak{A}$ is recursive, that the relations $\leq_{\beta}$ for $1 \leq \beta<\alpha$ are uniformly r.e. when indexed by $\left\{b: b<_{0} a\right\}$ for 
some $a \in \mathcal{O}$ where $|a|=\alpha$ and that the relation $x \in \operatorname{cl}_{\alpha}(\bar{p})$ is $\Delta_{2}^{0}$. Then $\mathfrak{A}$ is $\Delta_{\alpha}^{0}$-stable iff $\mathfrak{A}$ has a formally $\Delta_{\alpha}^{0}$ enumeration.

Similarly, Theorems 2 and 3 give

THEOREM 5. Suppose that the existential diagram of $\mathfrak{A}$ is recursive, that the relations $\leq_{\beta}$ for $1 \leq \beta<\alpha$ are uniformly r.e., and that the relations $x \in \operatorname{cl}_{\beta}(\bar{p})$ for $\beta<\alpha$ are uniformly $\Delta_{2}^{0}$. Then $\mathfrak{A}$ is $\hat{\Delta}^{0}$-stable iff $\mathfrak{A}$ has a formally $\Delta_{\beta}^{0}$ enumeration for some $\beta<\alpha$.

This establishes the following result, involving only the notions of $\S 2$.

COROLlaRY. Under the assumptions of Theorem $5, \mathfrak{A}$ is $\hat{\Delta}_{\alpha}^{0}$-stable iff $\mathfrak{A}$ is $\Delta_{\beta}^{0}$-stable for some $\beta<\alpha$.

Another re-statement of these results concerns the Scott rank of a structure $\mathfrak{A}$. For sequences $\bar{a}$ and $\bar{b}$ of the same length from $A$, define $\bar{a} \sim \bar{b}$ if there is an automorphism of $\mathfrak{A}$ which maps each entry of $\bar{a}$ to the corresponding entry of $\bar{b}$. From several slight variations, we choose to define the rank of $\mathfrak{A}$ to be the least ordinal $\alpha$ for which, for each $\bar{a} \in A$, there exists $\bar{c} \in A$ and $\beta<\alpha$ such that, for all $\bar{b}, \bar{d}$ of the same lengths as $\bar{a}, \bar{c}$, if $\bar{a}, \bar{c} \leq_{\beta} \bar{b}, \bar{d}$, then $\bar{a} \sim \bar{b}$. Equivalently, the rank is the least ordinal $\alpha$ such that, for each $\bar{a} \in A$, there is a $\Sigma_{\alpha}$ formula $\theta$ such that $\bar{b} \sim \bar{a}$ iff $\mathfrak{A} \vDash \theta[\bar{b}]$. If the rank of $\mathfrak{A}$ is $\alpha$, then there exists a $\Pi_{\alpha+1}$ sentence $\psi$ such that for each countable $\mathfrak{B}, \mathfrak{B} \cong \mathfrak{A}$ iff $\mathfrak{B} \vDash \psi$.

In the presence of our other assumptions a recursive structure $\mathfrak{A}$ has a formally $\Delta_{\alpha}^{0}$ enumeration iff, for some $\bar{p}$, the structure $(\mathfrak{A}, \bar{p})$ has rank at most $\alpha$ and is rigid, that is, has no automorphism other than the identity function. Thus we have:

THEOREM 6. Under the assumptions of Theorem $4, \mathfrak{A}$ is $\Delta_{\alpha}^{0}$-stable iff, for some finite sequence $\bar{p}$ from $A,(\mathfrak{A}, \bar{p})$ is rigid and has rank at most $\alpha$.

Similarly, under the assumptions of Theorem $5, \mathfrak{A}$ is $\hat{\Delta}_{\alpha}^{0}$-stable iff, for some finite sequence $\bar{p}$ from $A,(\mathfrak{A}, \bar{p})$ is rigid and has rank strictly below $\alpha$.

COMMENT. The statement of Theorem 6 could be varied using the facts that if $\mathfrak{A}$ has $2^{\aleph_{0}}$ automorphisms then so has each $(\mathfrak{A}, \bar{p})$, while if $\mathfrak{A}$ has fewer than $2^{\aleph_{0}}$ automorphisms then, for some $\bar{p},(\mathfrak{A}, \bar{p})$ is rigid.

HYP-STABILITY. A final notion along the lines of this paper would be to define the recursive structure $\mathfrak{A}$ to be $H y p$-stable if, for each recursive $\mathfrak{B} \cong \mathfrak{A}$, every isomorphism from $\mathfrak{B}$ to $\mathfrak{A}$ is hyperarithmetical, that is, $\Delta_{\alpha}^{0}$-recursive for some $\alpha<\omega_{1}^{C K}$. However, this matter is quickly disposed of, using the results from [4] that the rank of a recursive structure is at most $\omega_{1}^{C K}$ and that if its rank is equal to $\omega_{1}^{C K}$, then the structure has $2^{\aleph_{0}}$ automorphisms.

Thus, if $\mathfrak{A}$ has rank $\omega_{1}^{C K}$, then $\mathfrak{A}$ is not Hyp-stable. Now suppose that the rank of $\mathfrak{A}$ is $\alpha<\omega_{1}^{C K}$. From the definition of rank, we have $\bar{a} \leq_{\alpha} \bar{b} \Rightarrow \bar{a} \sim \bar{b}$. Without further assumptions, we do not have the conclusion of Lemma 6 . However, we can show instead, by recursive transfinite induction, that, uniformly in $\bar{a} \in A$ and a notation for a limit ordinal $\delta$, one can find a Gödel number for a recursive $\Pi_{\delta+2 n}$ formula $\phi$ such that $\bar{a} \leq_{\delta+n} \bar{b}$ iff $\mathfrak{A} \vDash \phi[\bar{b}]$. Thus, if $(\mathfrak{A}, \bar{p})$ is rigid, then $\mathfrak{A}$ has a formally $\Delta_{\delta+2 n+1}^{0}$ enumeration, and so by Theorem 3 is $\Delta_{\delta+2 n+1}^{0}$-stable. We have shown the following theorem. 
THEOREM 7. A recursive structure $\mathfrak{A}$ is Hyp-stable iff $\mathfrak{A}$ has fewer than $2^{\aleph_{0}}$ automorphisms. In this case, if the rank of $\mathfrak{A}$ is $\delta+n$, where $\delta$ is a limit ordinal or zero, then $\mathfrak{A}$ is $\Delta_{\delta+2 n+1}^{0}$-stable.

4. Examples. For many examples of recursive algebraic structures, such as Boolean algebras and abelian groups, the relations $\leq_{\alpha}$ can be shown to be uniformly recursive. However, those examples of this kind which we have considered and which have infinite rank also have $2^{\aleph_{0}}$ automorphisms and so do not provide examples of $\Delta_{\alpha}^{0}$-stable structures for $\alpha$ infinite. [They do provide examples of $\Delta_{\alpha}^{0}$-categorical strucrtures, but this is not our present topic.] We may, however, illustrate the notions of this paper by considering briefly the structures $(\alpha,<)$ and their expansions. These will always be rigid and have arbitrarily large ranks.

We make the following definition.

Definition. A recursive structure is strictly $\Delta_{\alpha}^{0}$-stable if it is $\Delta_{\alpha}^{0}$-stable and not $\Delta_{\beta}^{0}$-stable for any $\beta<\alpha$.

We comment that this notion depends only on the classical isomorphism type of the structure, and that in view of Theorem 7 , every recursive structure with fewer than $2^{\aleph_{0}}$ isomorphisms is strictly $\Delta_{\alpha}^{0}$-stable for some unique $\alpha<\omega_{1}^{C K}$. In all our examples, by Theorem 2 and its consequences, the structures which are strictly $\Delta_{\alpha}^{0}$-stable for some limit ordinal $\alpha$ are also not $\hat{\Delta}_{\alpha}^{0}$-stable.

In what follows, $\delta$ will always denote a limit ordinal or zero and $n$ will denote a finite ordinal.

EXAMPLE 1. In the case where $\mathfrak{A}=(\alpha,<)$ for some ordinal number $\alpha$, we note that, if $\bar{a} \leq_{\gamma} \bar{b}$ for $\gamma \geq 1$, then the elements of the sequence $\bar{a}$ will be in the same order as those of $\bar{b}$. We need therefore consider only the case where $\bar{a}=a_{1}, a_{2}, \ldots, a_{n}, a_{1} \leq a_{2} \leq \cdots \leq a_{n}, \bar{b}=b_{1}, b_{2}, \ldots, b_{n}$, and $b_{1} \leq b_{2} \leq \cdots \leq b_{n}$, and we may easily see that the relation $\bar{a} \leq_{\gamma} \bar{b}$ depends only on the order types of the intervals $\left[0, a_{1}\right),\left[a_{1}, a_{2}\right), \ldots,\left[a_{n}, \infty\right)$, and those of the corresponding intervals for $\bar{b}$.

So we introduce the corresponding relations $\leq_{\gamma}$ between ordinal numbers, including 0 , and so between well-ordered sets. Let $\alpha \leq_{1} \beta$ if $\alpha=\beta=0$ or if $\alpha$ is infinite and $\beta>0$ or if $\alpha$ is finite and $0<\beta \leq \alpha$. Define $\alpha \leq_{\gamma+1} \beta$ if, whenever $\beta=\beta_{0}+1+\beta_{1}+1+\cdots+1+\beta_{m}$, then there exist $\alpha_{i}$ such that $\alpha=\alpha_{0}+1+\alpha_{1}+1+\cdots+1+\alpha_{m}$, and $\alpha_{i} \geq_{\gamma} \beta_{i}$ for $0 \leq i \leq m$. Define $\alpha \leq_{\delta} \beta$ to hold for a limit ordinal $\delta$ if $\alpha \leq_{\gamma} \beta$ for all $\gamma<\delta$.

Now we may show, by transfinite induction on $\gamma$, that if $a_{1} \leq a_{2} \leq \cdots \leq a_{n}$ and $b_{1} \leq b_{2} \leq \cdots \leq b_{n}$, then $\bar{a} \leq_{\gamma} \bar{b}$ iff $\left[0, a_{1}\right) \leq_{\gamma}\left[0, b_{1}\right),\left[a_{n}, \infty\right) \leq_{\gamma}\left[b_{n}, \infty\right)$ and for $1 \leq i<n,\left[a_{i}, a_{i+1}\right) \leq_{\gamma}\left[b_{i}, b_{i+1}\right)$.

It remains to find the conditions for which $\alpha \leq_{\gamma} \beta$, which may be verified by transfinite induction. We note that each $\alpha$ may be written as a finite decreasing sum $\alpha=\sum_{\xi} \omega^{\xi} \cdot m_{\xi}$ where each $m_{\xi}<\omega$ and also, for each $\xi$, we may put $\alpha=\omega^{\xi} \cdot \alpha_{\xi}+\rho_{\xi}$ where $\rho_{\xi}<\omega^{\xi}$. [Thus $\omega^{\xi} \cdot \alpha_{\xi}=\sum_{\eta \geq \xi} \omega^{\eta} \cdot m_{\eta}$ and $\rho_{\xi}=\sum_{\eta<\xi} \omega^{\eta} \cdot m_{\eta}$, with the sums again taken in decreasing order.] Similarly, let $\beta=\sum \omega^{\xi} \cdot n_{\xi}$ and for each $\xi$ let $\beta=\omega^{\xi} \cdot \beta_{\xi}+\sigma_{\xi}$ where each $n_{\xi}<\omega$ and each $\sigma_{\xi}<\omega^{\xi}$.

LEMMA 7. (i) If $\gamma=\delta+2 n+1$, then $\alpha \leq_{\gamma} \beta$ iff one of the following conditions hold. 
(a) $\alpha=\beta$, (b) $\rho_{\delta+n}=\sigma_{\delta+n}, \alpha_{\delta+n+1} \geq 1$, and $\beta_{\delta+n} \neq 0$, (c) $\rho_{\sigma+n}=\sigma_{\delta+n}$, $\alpha_{\delta+n+1}=\beta_{\delta+n+1}=0$, and $m_{\delta+n} \geq n_{\delta+n}>0$.

(ii) If $\gamma=\delta+2 n+2$, then $\alpha \leq_{\gamma} \beta$ iff either

(a) $\alpha=\beta$, or (b) $\rho_{\delta+n}=\sigma_{\delta+n}, \alpha_{\delta+n+1} \geq 1, \beta_{\delta+n+1} \geq 1$, and $m_{\delta+n} \geq n_{\delta+n}$.

(iii) If $\gamma=\delta \neq 0$, then $\alpha \leq_{\gamma} \beta$ iff either

(a) $\alpha=\beta$, or (b) $\rho_{\delta}=\sigma_{\delta}, \alpha_{\delta} \geq 1$, and $\beta_{\delta} \geq 1$.

To use Theorems 1 and 2, we must also examine the sets $\operatorname{cl}_{\gamma}(\bar{a})$. It is sufficient to consider only the cases where $\alpha$ is of the form $\omega^{\xi}$. Then from Lemma 7 we may show the following.

LEMMA 8. (i) In $\left(\omega^{\delta+n},<\right)$ for $n \geq 1, \delta+n \geq 2$ we have $\mathrm{cl}_{\delta+2 n}(\varnothing)=\omega^{\delta+n}$ while, for all $\bar{a}, \max \left\{a_{i}\right\}+\omega^{\delta+n-1} \notin \mathrm{cl}_{\delta+2 n-1}(\bar{a})$.

(ii) In $\left(\omega^{\delta},<\right)$ for $\delta \neq 0$ we have $\operatorname{cl}_{\delta}(\varnothing)=\omega^{\delta}$ while, for all $\bar{a}$ and all $\gamma<\delta$, we have, for example, $\max \left\{a_{i}\right\}+\omega^{\gamma} \notin \mathrm{cl}_{\gamma}(\bar{a})$.

Thus, from (i), if $\delta<\omega_{1}^{C K}$ then by Lemma $6\left(\omega^{\delta+n},<\right)$ has a formally $\Delta_{\delta+2 n}^{0}$ enumeration and so is $\Delta_{\delta+2 n}^{0}$-stable. But, also from (i), for each $\bar{p}, \omega^{\delta+n} \neq$ $\operatorname{cl}_{\delta+2 n-1}(\bar{p})$, which will show that $\left(\omega^{\delta+n},<\right)$ is not $\Delta_{\delta+2 n-1}^{0}$-stable provided that the conditions of Theorem 1 can be satisfied. This presents no difficulty since for each notation $a \in \mathcal{O}$ for $\alpha<\omega_{1}^{C K}$ we may convert $\left\{b \in 0: b<_{0} a\right\}$ to a recursive well-ordering of type $\omega^{\alpha}$ by the process of exponentiation. That is, we let each sequence $b_{1}, \ldots, b_{n}$ for which $a>_{0} \quad b_{1} \geq_{0} b_{2} \geq_{0} \cdots \geq_{0} b_{n}$ represent the ordinal $\omega^{\beta_{1}}+\omega^{\beta_{2}}+\cdots+\omega^{\beta_{n}}$ where $\beta_{i}=\left|b_{i}\right|$. For this ordering it is clear from Lemma 7 that each relation $\leq_{\gamma}$ is recursive, uniformly in the notation $c<_{0} a$ for $\gamma$. Thus condition (2) and similarly, by Lemma 8(i), condition (3) of Theorem 1 are satisfied. Condition (1) is also satisfied since, in this ordering, the successor relation is recursive.

In the same fashion, from Lemma 7 and Lemma 8(ii), we see that $\left(\omega^{\delta},<\right)$ is $\Delta_{\delta}^{0}$-stable but not $\hat{\Delta}_{\delta}^{0}$-stable. Ordinals not of the form $\omega^{\xi}$ are nevertheless finite sums of such ordinals and each summand can be treated separately. So we obtain the following.

THEOREM 8. If $\omega^{\delta+n} \leq \alpha<\omega^{\delta+n+1}$ where $\delta+n \leq 2$, then $(\alpha,<)$ is strictly $\Delta_{\delta+2 n}^{0}$-stable. $[\mathbf{1}]$.

The result also holds for $\delta+n=1$, using Goncharov's Theorem, described in

EXAMPLE 2. For structures of the form $(\alpha,<, S)$ where $S$ is the successor relation, several shortcuts are possible. For example, by arguing in terms of formulae, we can show that $\bar{a} \leq_{\gamma} \bar{b}$ in $(\alpha,<, S)$ iff $\bar{a} \leq_{1+\gamma} \bar{b}$ in $(\alpha,<)$. So using the methods of Example 1 we have

THEOREM 9. If $\omega^{1+\delta+n} \leq \alpha<\omega^{1+\delta+n+1}$ where $\delta+n \geq 0$, then $(\alpha,<, S)$ is strictly $\Delta_{1+\delta+2 n}^{0}$-stable.

EXAMPLE 3. Similarly, for structures $(\alpha,<, S, L)$ where $L$ is the set of limit ordinals, we have $\bar{a} \leq_{\gamma} \bar{b}$ in $(\alpha,<, S, L)$ iff $\bar{a} \leq_{2+\gamma} \bar{b}$ in $(\alpha,<)$.

This leads to the following theorem. 
THEOREM 10. If $\omega^{1+\delta+n} \leq \alpha<\omega^{1+\delta+n+1}$ where $\delta+n \geq 1$, then $(\alpha,<, S, L)$ is strictly $\Delta_{\delta+2 n}^{0}$-stable. [For $\alpha<\omega^{2},(\alpha,<, S, L)$ is $\Delta_{1}^{0}$-stable.]

EXAMPLE 4. For structures of the form $(\alpha,<,+)$, it is clearly preferable to proceed indirectly. We may first [by careful economy with quantifiers] show that in such a structure the set $P_{\xi}$ of ordinals of the form $\omega^{\omega^{\xi} \cdot \beta}$ is given for each $\xi$ by an infinitary recursive $\Pi_{\delta+2 n}^{0}$ formula, where $\xi=\delta+n$ [except that $P_{0}$ is $\Pi_{1}^{0}$ ] and that the successor relation $S_{\xi}$ relative to $P_{\xi}$ is given by a recursive $\Pi_{\delta+2 n+1}$ formula. This shows that the structure $\left(\omega^{\omega^{\delta+n+1}},<,+\right)$ has a formally $\Delta_{\delta+2 n+2}^{0}$ enumeration and so is $\Delta_{\delta+2 n+2^{-} \text {-stable. }}^{0}$

To show that this structure is strictly $\Delta_{\delta+2 n+2}^{0}$-stable, we may use Example 1 . Since $\omega^{\delta+n+1}$ is strictly $\Delta_{\delta+2 n+2}^{0}$-stable, there exist recursive orderings of type $\omega^{\delta+n+1}$ which are not $\Delta_{\delta+2 n+1}^{0}$ isomorphic. As in Example 1, these may be converted by exponentiation to structures of isomorphism type $\left(\omega^{\omega^{6+2 n+1}},<,+\right)$, and these structures cannot be $\Delta_{\delta+2 n+1}^{0}$-isomorphic. [Note, however, that this transformation may be used only in this direction, since in a recursive structure of type $(\alpha,<,+)$, the elements corresponding to the ordinals $\omega^{\xi}$ may not be recursive.] By similar arguments we have the following.

THEOREM 11. If $\omega^{\delta+n} \leq \alpha<\omega^{\delta+n+1}$ where $\delta+n \geq 1$, then $\left(\omega^{\alpha},<,+\right)$ is strictly $\Delta_{\delta+2 n}^{0}$-stable. [For $\alpha<\omega,\left(\omega^{\alpha},<,+\right)$ is $\Delta_{1}^{0}$-stable. $]$

EXAMPLE 5. For structures of the form $(\alpha,<,+, \cdot)$, the existential diagram will not be recursive, so Theorems 1 and 2 as given will certainly not apply. We may, however, follow Example 4 and show that $\left(\omega^{\omega^{\omega^{\delta+n}}},<,+, \cdot\right)$ has a formally $\Delta_{\delta+2 n}^{0}$ enumeration and then apply the same exponentiation construction again, using the fact that $\left(\omega^{\omega^{\delta+n}},<,+\right)$ is strictly $\Delta_{\delta+2 n}^{0}$-stable, to show the following.

THEOREM 12. If $\omega^{\delta+n} \leq \alpha<\omega^{\delta+n+1}$, where $\delta+n \geq 1$, then $\left(\omega^{\omega^{\alpha}},<,+, \cdot\right)$ is strictly $\Delta_{\delta+2 n}^{0}$-stable. [For $\alpha<\omega,\left(\omega^{\omega^{\alpha}},<,+, \cdot\right)$ is $\Delta_{1}^{0}$-stable.]

5. Conclusion. The equivalence of $\Delta_{\alpha}^{0}$-stability to the existence of a formally $\Delta_{\alpha}^{0}$ enumeration does seem to require further assumptions. A counter-example along these lines for $\alpha=1$ was given by Goncharov in [5]. The author does not know at this stage whether the conditions of Theorems 1 and 2 can be significantly weakened. Certainly there are many counter-examples yet to be constructed.

The constructions of $\S 1 \mathrm{can}$ also be applied to the notion of $\Delta_{\alpha}^{0}$-categoricity. These results will be given in $[\mathbf{2}]$. E. Barker has obtained results concerning the topic of "intrinsically $\Sigma_{\alpha}^{0}$ " relations, which will be given in $[\mathbf{3}]$.

\section{REFERENCES}

1. C. J. Ash, Stability of recursive structures in arithmetical degrees, Ann. Pure Appl. Logic (to appear).

2. Categoricity of recursive structures in hyperarithmetical degrees (in preparation).

3. E. Barker, Intrinsically $\Sigma_{\alpha}^{0}$ relations (preprint).

4. J. Barwise, Admissible sets and structures, Springer-Verlag, Berlin and New York, 1975.

5. S. S. Goncharov, The quantity of nonautoequivalent constructivizations, Algebra and Logic 16 (1977), 169-185.

6. H. Rogers, Theory of recursive functions and effective computability, McGraw-Hill, New York, 1967.

Department of Mathematics, Monash University, Clayton, Victoria 3168, 